\title{
Microinhomogeneity for Aqueous Mixtures of Water-miscible Organic Solvents
}

\author{
Toshiyuki TAKAMUKU, ${ }^{\dagger}$ Yasukuni NOGUCHI, Miki NAKANO, Masaru MATSUGAMI,* \\ Hiroki IWASE** and Toshiya OTOMO*** \\ Department of Chemistry and Applied Chemistry, Faculty of Science and Engineering, Saga University, Honjo-machi, Saga 840-8502 \\ *Department of General Education, Kumamoto National College of Technology, 2659-2, Suya, Koshi, Kumamoto 861-1102 \\ **Quantum Beam Science Directorate, Japan Atomic Energy Agency (JAEA), Tokai, Ibaraki 319-1195 \\ ***Institute of Materials Structure Science, High Energy Accelerator Research Organization (KEK), Oho, Tsukuba 305-0801
}

\begin{abstract}
Mixing states for aqueous solutions of water-miscible organic solvents, acetonitrile and 1,4-dioxane, have been investigated at the molecular level by means of small-angle neutron scattering (SANS) and NMR relaxation. It has been shown that the organic solvent and water molecules are inhomogeneously mixed each other at the molecular level, although their mixtures are homogeneous at the macroscopic scale. Thus, both organic solvent clusters and water clusters coexist in the mixtures. The present investigation has also revealed that the microinhomogeneity of acetonitrile-water and 1,4-dioxane-water mixtures is gradually enhanced by cooling or adding salts toward phase separation. Based on the results, the effect of microinhomogeneity for solvent mixtures on chemical reactions was considered.

[Received August 31, 2007; Accepted September 20, 2007]
\end{abstract}

Key-words : Mixing state, Aqueous mixtures, Clusters, Phase separation, SANS, NMR relaxation

\section{Introduction}

Various organic solvents, such as aliphatic alcohols, acetonitrile (AN), and 1,4-dioxane (dio), are miscible with water at any ratio, and aqueous mixtures of the water-miscible organic solvents have often been utilized as a reaction field of chemical synthesis and solvent extraction. However, solvent mixtures used as a reaction field are often regarded as a continuous medium, and the combination and mixing ratio of both solvents are decided in a trial-and-error manner. On the other hand, so far, the inhomogeneous mixing of binary solutions has been discussed on the basis of various thermodynamic parameters, such as partial molar volume and enthalpy of mixing. ${ }^{1), 2)}$

The development of experimental techniques has enabled us to clarify liquid structure of pure solvents and mixing state of binary solutions at the molecular level. X-ray and neutron scattering techniques are the most powerful tools to directly observe static structure of pure solvents and solvent mixtures. Large-angle scattering techniques with X-ray and neutron give us information on liquid structure at the microscopic scale in terms of interatomic distance, coordination number, and temperature factor. On the other hand, small-angle scattering techniques make clear the concentration fluctuations of solvent mixtures, i.e., cluster formation in solvent mixtures can be observed at the mesoscopic scale. In particular, small-angle neutron scattering (SANS) experiments with H/D isotope substitution method can highly contrast clusters of deuterated solvents with those of undeuterated ones due to the larger neutron scattering length $(6.67 \mathrm{fm})$ of $\mathrm{D}$ atom than that $(-3.74$ $\mathrm{fm})$ of $\mathrm{H}$ atom. ${ }^{3)}$ Moreover, dynamics of solvent molecules, such as translational and rotational motions, can be elucidated by using NMR relaxation technique. For instance, the rotational motion of $\mathrm{D}_{2} \mathrm{O}$ molecules in heavy water mixtures with organic solvents can be observed by using ${ }^{2} \mathrm{H}$ NMR relaxation measurements. ${ }^{4}$ )

Here, the mixing states of acetonitrile-water and 1,4-dioxane-water mixtures have been clarified by using SANS technique. In addition, the change in the mixing states of both mixtures by cooling or addition of salts toward phase separation has been observed by means of SANS and NMR relaxa-

\footnotetext{
${ }^{\dagger}$ Corresponding author; E-mail address: takamut@cc.saga-u.ac.jp
}

tion. On the basis of the present results, the microinhomogeneity of the organic solvent-water mixtures is discussed at the molecular level. Moreover, the effect of microinhomogeneity for solvent mixtures on chemical synthesis and reaction is considered.

\section{Experiments}

2.1 Chemical reagents

Acetonitrile (Wako Pure Chemicals, extra grade) was dried on thermally activated $0.4-\mathrm{nm}$ molecular sieves for several days, and then, distilled under nitrogen atmosphere. 1,4Dioxane (Wako Pure Chemicals, extra grade) was refluxed with metal sodium wire for $8 \mathrm{~h}$, and then distilled under the atmospheric pressure. Heavy water $\left(\mathrm{D}_{2} \mathrm{O}\right)$ (Aldrich, D atom content of $99.9 \%$ ) was used without further purification. Lithium chloride, sodium chloride, and potassium chloride (Wako Pure Chemicals, extra grade) were dried at $393 \mathrm{~K}$ with an electric oven for $6 \mathrm{~h}$ before use. Organic solvent- $\mathrm{D}_{2} \mathrm{O}$ binary mixtures were prepared by weighing heavy water and distilled organic solvents to the required mole fractions of organic solvent under dry nitrogen gas atmosphere in a glove box. Acetonitrile- $\mathrm{D}_{2} \mathrm{O}-\mathrm{MCl}\left(\mathrm{M}=\mathrm{Li}^{+}, \mathrm{Na}^{+}\right.$, and $\left.\mathrm{K}^{+}\right)$and 1,4dioxane- $\mathrm{D}_{2} \mathrm{O}-\mathrm{NaCl}$ ternary solutions were prepared by weighing distilled organic solvents and heavy water $\mathrm{MCl}$ solutions at the required $\mathrm{MCl}$ concentrations to reach acetonitrile and 1,4dioxane mole fractions of $x_{\mathrm{AN}}=0.25$ and $x_{\mathrm{dio}}=0.17$, respectively, which correspond to the volume ratio of the organic solvent to water of $1: 1$. Densities for the sample solutions were measured by using a densimeter (ANTON Paar K.G., DMA60 and DMA602).

\subsection{SANS experiments}

SANS intensities for acetonitrile- $\mathrm{D}_{2} \mathrm{O}$ mixtures at $x_{\mathrm{AN}}=0.2$, $0.25,0.3,0.4,0.5$, and 0.6 were measured as a function of temperatures in the range from 279 to $307 \mathrm{~K}$ on SWAN spectrometer at a pulsed neutron facility (KENS) in High Energy Accelerator Research Organization (KEK), Tsukuba, Japan. Those for acetonitrile- $\mathrm{D}_{2} \mathrm{O}-\mathrm{MCl}$ mixtures at $x_{\mathrm{AN}}=0.25$ were also measured at $298 \mathrm{~K}$ on the spectrometer. The SWAN covers the momentum transfer $Q\left(=4 \pi \lambda^{-1} \sin \theta\right)$ range of $0.1-120 \mathrm{~nm}^{-1} .^{5)}$ The sample solutions were kept in a quartz cell of $22 \mathrm{~mm}$ in width, $25 \mathrm{~mm}$ in height, and $2 \mathrm{~mm}$ in sample thickness. The sample temperature was controlled within 

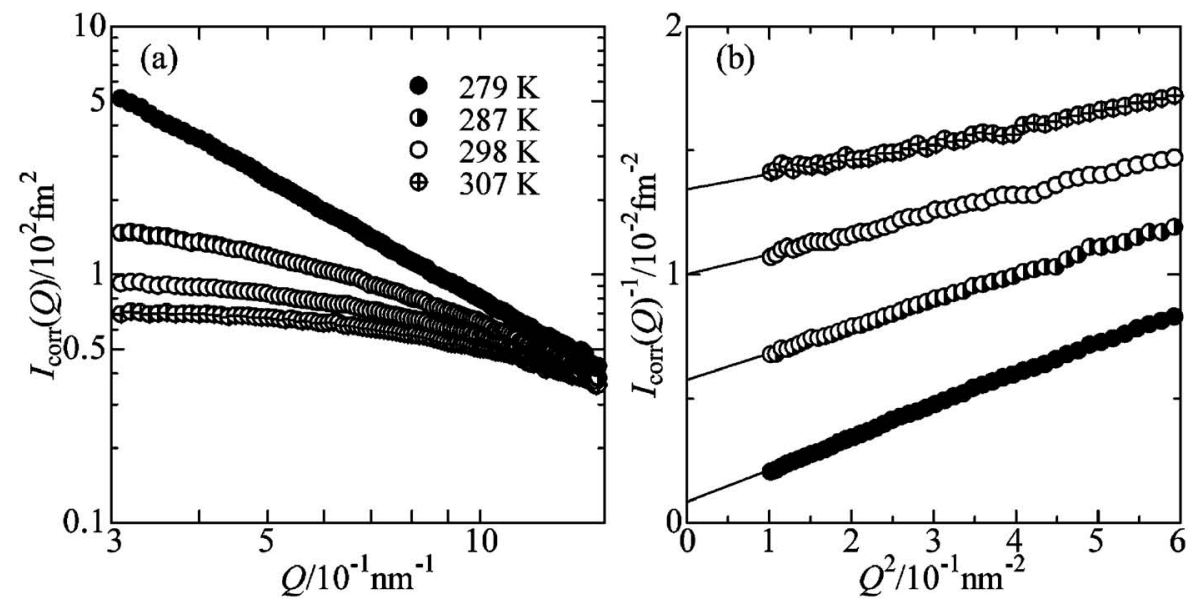

Fig. 1. (a) Normalized SANS intensities for acetonitrile- $\mathrm{D}_{2} \mathrm{O}$ mixtures at 279, 287, 298, and $307 \mathrm{~K}$ when $x_{\mathrm{AN}}=0.4$ and (b) Ornstein-Zernike plots. ${ }^{10)}$ Circles give the experimental values and solid lines the theoretical ones.

$\pm 0.5 \mathrm{~K}$ by circulation of temperature-controlled silicone oil in a sample jacket. The neutron beam size at a sample position was $20 \times 20 \mathrm{~mm}^{2}$, and the wavelength range used was $\lambda=$ $0.05-1.16 \mathrm{~nm}$. The scattering intensities were accumulated for $4 \mathrm{~h}$ per sample. The observed intensities were corrected for background, absorption, and cell scattering. The transmission by a sample and a cell was measured with a ${ }^{3} \mathrm{He}$ position sensitive detector placed at a beam stopper position. The correction for detector efficiencies and the normalization to absolute units were made by dividing the intensities of each solution by those of vanadium. ${ }^{5)}$

SANS intensities for 1,4-dioxane- $\mathrm{D}_{2} \mathrm{O}-\mathrm{NaCl}$ mixtures at $x_{\text {dio }}$ $=0.17$ and various $\mathrm{NaCl}$ concentrations were measured on WINK spectrometer at KENS of KEK. The details of WINK spectrometer has been described elsewhere. ${ }^{6)}$ A sample solution was kept in a quartz cell of $22 \mathrm{~mm}$ in width, $40 \mathrm{~mm}$ in height, and $2 \mathrm{~mm}$ in sample thickness, and placed in a vacuum chamber at $296.2 \pm 0.5 \mathrm{~K}$ during measurements. SANS data were collected on the WINK for 6-9 h for each sample solution. The measurements were also made on $\mathrm{H}_{2} \mathrm{O}$ in a quartz cell of $1 \mathrm{~mm}$ in sample thickness as a standard material, an empty cell, and background. Corrections for background, absorption, cell scattering, and detector efficiencies and normalization to absolute units, were made as described in the previous paper. ${ }^{7)}$

The incoherent scattering was subtracted from the normalized intensities obtained on both SWAN and WINK. All parameter values required for the above corrections were taken from the literature. ${ }^{8)}$

\subsection{NMR relaxation experiments}

${ }^{2} \mathrm{H}$ spin-lattice relaxation times $T_{1}$ of $\mathrm{D}_{2} \mathrm{O}$ molecules in pure $\mathrm{D}_{2} \mathrm{O}$ and acetonitrile- $\mathrm{D}_{2} \mathrm{O}$ mixtures at $x_{\mathrm{AN}}=0.1,0.2,0.25,0.3$, $0.4,0.5,0.6,0.7$, and 0.8 were measured at $278,288,298$, and $308 \mathrm{~K}$ by using an FT-NMR spectrometer (JEOL, JNMAL300). The sample solutions was kept in a 5-mm sample tube (Shigemi, PS-003), and the probe temperature was controlled within $\pm 0.1 \mathrm{~K}$ by a mixture of hot air and a dry nitrogen stream from liquid nitrogen. $T_{1}$ was measured by an inversion-recovery method with a pulse sequence $(\pi-\tau-\pi / 2)^{n}$, where the number $n$ of the delay times $\tau$ in the series of the sequence was 13 for the present experiments. The longest delay time exceeded $5 T_{1}$. The $T_{1}$ data were measured four or five times for each sample solution and averaged to obtain a final value. The observed frequency range was $500 \mathrm{~Hz}$; sam- pling points were 2,048; the digital resolution was $0.488 \mathrm{~Hz}$.

\section{Results and discussion}

\subsection{Acetonitrile- $\mathrm{D}_{2} \mathrm{O}$ mixtures}

SANS intensities of the acetonitrile- $\mathrm{D}_{2} \mathrm{O}$ mixtures in the acetonitrile mole fraction range from $x_{\mathrm{AN}}=0.2$ to 0.6 were measured as a function of temperature from 279 to $\left.307 \mathrm{~K}^{9}{ }^{9)}, 10\right)$ In Fig. 1 (a), for example, the normalized SANS intensities for the mixture at $x_{\mathrm{AN}}=0.4$ are depicted as a function of temperatures. The SANS intensities are significantly observed even at 307 and $298 \mathrm{~K}$ and further strengthened when the temperature decreases. This result suggests that acetonitrile and water molecules are inhomogeneously mixed at the molecular level, though both solvents are homogeneously mixed at the macroscopic scale. The microinhomogeneity of the mixture is enhanced with lowering temperature. As seen in Fig. 1(b), to quantitatively elucidate the microinhomogeneity of the mixture, the Ornstein-Zernike fits were made on the reciprocal SANS intensities as a function of the $Q^{2}$ through the equation of $I_{\text {corr }}(Q)^{-1}=I(0)^{-1}\left[1+\xi^{2} Q^{2}\right]$. The $\xi$ represents the Ornstein-Zernike correlation length, ${ }^{11)}$ corresponding to the size of clusters formed in the mixture. The theoretical values (solid lines) estimated by a least-squares fit with using the equation well explain the observed ones (circles). The same analysis was made on the SANS intensities for the mixtures at the other mole fractions studied. ${ }^{10}$ )

In Fig. 2, the $\xi$ values for the mixtures at each temperature are depicted as a function of $x_{\mathrm{AN}}$. At $298 \mathrm{~K}$ the $\xi$ values for the mixtures at $x_{\mathrm{AN}}=0.3$ and 0.4 are larger than those at the other mole fractions. This shows that acetonitrile and water molecules are most inhomogeneously mixed each other at $x_{\mathrm{AN}}=0.3$ and 0.4 in the mole fraction range studied. Thus, both acetonitrile clusters and water clusters are significantly formed in the mixtures at the mole fractions. When the temperature decreases from 307 to $279 \mathrm{~K}$, the $\xi$ values for the mixtures at $x_{\mathrm{AN}}=0.3$ and 0.4 drastically increase, while the increase in the $\xi$ values at $x_{\mathrm{AN}}=0.2$ and 0.6 is not remarkable. It is shown that both clusters are most evolved in the acetonitrile-water mixture between $x_{\mathrm{AN}}=0.3$ and 0.4 with lowering temperature. This finding is reasonable with the fact that the upper critical solution temperature (UCST) is found at $272 \mathrm{~K}$ and $x_{\mathrm{AN}}=$ 0.38 , i.e., acetonitrile-water mixtures are separated into acetonitrile-rich and water-rich phases by cooling. ${ }^{12)}$ It is thus concluded that both clusters observed at the higher tempera- 


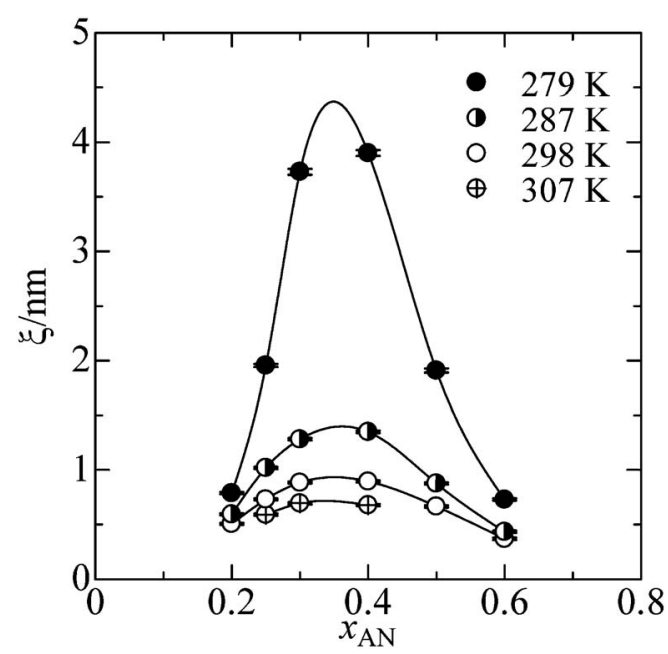

Fig. 2. Correlation lengths $\xi$ with the standard deviations $\sigma$ as error bars for acetonitrile- $\mathrm{D}_{2} \mathrm{O}$ mixtures at $279,287,298$, and $307 \mathrm{~K}$ as a function of $x_{\mathrm{AN}} \cdot{ }^{10)}$

Table 1. ${ }^{2} \mathrm{H}$ Spin-Lattice Relaxation Times $T_{1} / \mathrm{ms}$ for $\mathrm{D}_{2} \mathrm{O}$ and Acetonitrile- $\mathrm{D}_{2} \mathrm{O}$ Mixtures at Various Temperatures

\begin{tabular}{lllll}
\hline$x_{\mathrm{AN}}$ & $278 \mathrm{~K}$ & $288 \mathrm{~K}$ & $298 \mathrm{~K}$ & $308 \mathrm{~K}$ \\
\hline 0 & $222(11)$ & $312(12)$ & $422(1)$ & $559(10)$ \\
0.1 & $188(1)$ & $270(5)$ & $371(5)$ & $489(3)$ \\
0.2 & $202(3)$ & $287(3)$ & $378(3)$ & $497(7)$ \\
0.25 & $208(4)$ & $294(3)$ & $389(3)$ & $498(9)$ \\
0.3 & $214(1)$ & $301(1)$ & $397(6)$ & $512(10)$ \\
0.4 & $234(9)$ & $320(4)$ & $417(2)$ & $538(7)$ \\
0.5 & $253(1)$ & $346(4)$ & $450(4)$ & $552(18)$ \\
0.6 & $291(13)$ & $377(2)$ & $482(17)$ & $608(3)$ \\
0.7 & $325(3)$ & $430(10)$ & $527(20)$ & $669(5)$ \\
0.8 & $409(17)$ & $519(1)$ & $645(14)$ & $771(31)$ \\
\hline
\end{tabular}

The values in parentheses are standard deviations for the last figure of $T_{1}$ values.

tures are a precursor of phase separation of the mixtures by cooling.

To clarify the mixing state of the binary solutions from the dynamics of water molecules, ${ }^{2} \mathrm{H}$ spin-lattice relaxation times $T_{1}$ for $\mathrm{D}_{2} \mathrm{O}$ molecules in pure $\mathrm{D}_{2} \mathrm{O}$ and the acetonitrile- $\mathrm{D}_{2} \mathrm{O}$ mixtures at $0.1 \leq x_{\mathrm{AN}} \leq 0.8$ were measured in the temperature range from 278 to $308 \mathrm{~K}$. In Table 1, the $T_{1}$ values determined for $\mathrm{D}_{2} \mathrm{O}$ and the acetonitrile- $\mathrm{D}_{2} \mathrm{O}$ mixtures are summarized. The $T_{1}$ of ${ }^{2} \mathrm{H}$ atom is governed mainly by a coupling of the electric quadrupole moment $e Q$ of the nucleus to the electric filed gradient $e q$. The spin-rotational and the intramolecular and intermolecular dipole-dipole contribution to the spin-lattice relaxation of ${ }^{2} \mathrm{H}$ can be neglected. Therefore, the ${ }^{2} \mathrm{H}-T_{1}$ of $\mathrm{D}_{2} \mathrm{O}$ gives information on the rotational motion of $\mathrm{D}_{2} \mathrm{O}$ molecules. The relation between the relaxation rate $R_{1}(=1 /$ $T_{1}$ ) of ${ }^{2} \mathrm{H}$ and the rotational correlation time $\tau_{2 \mathrm{R}}$ can be expressed by Eq. (1), under an extreme narrowing condition $\left(\omega \tau_{2 \mathrm{R}} \ll 1 ; \omega\right.$ is the resonance frequency $){ }^{4)}$

$$
R_{1}=\frac{3 \pi^{2}}{10}\left(\frac{e^{2} Q q}{h}\right)^{2} \frac{2 I+3}{I^{2}(2 I-1)}\left(1+\frac{\eta^{2}}{3}\right) \tau_{2 \mathrm{R}}
$$

where $e^{2} Q q / h$ is the quadrupole coupling constant, $\eta$ is the asymmetric parameter, and $I$ is the nuclear spin moment $(I=1$ for $\left.{ }^{2} \mathrm{H}\right)$. The correlation time $\tau_{2 \mathrm{R}}$ is a time-averaged value of

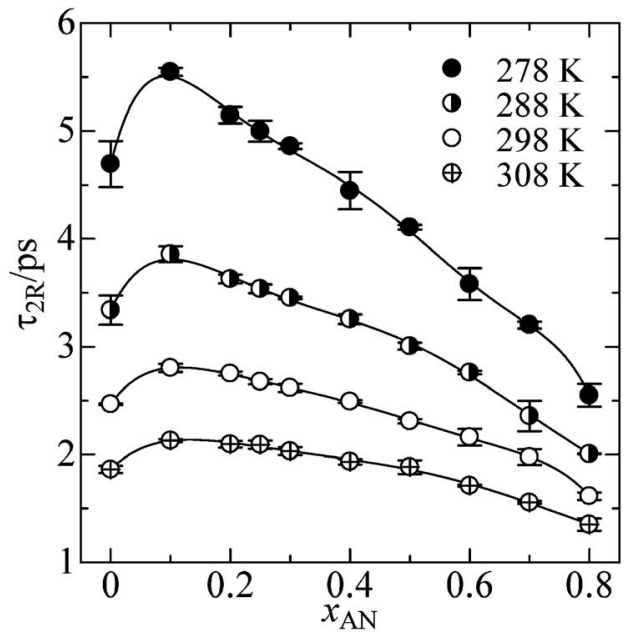

Fig. 3. Rotational correlation times $\tau_{2 \mathrm{R}}$ with the standard deviations $\sigma$ as error bars for acetonitrile- $\mathrm{D}_{2} \mathrm{O}$ mixtures at 278, 288, 298, and $308 \mathrm{~K}$ as a function of $x_{\mathrm{AN}}$.

the time correlation function of the second-order orientation of the O-D axis of a $\mathrm{D}_{2} \mathrm{O}$ molecule. To our knowledge, there are no reports on both $e^{2} Q q / h$ and $\eta$ values of ${ }^{2} \mathrm{H}$ in acetonitrile- $\mathrm{D}_{2} \mathrm{O}$ mixtures as a function of $x_{\mathrm{AN}}$ and temperature. In the present investigation, thus, reliable values determined for pure $\mathrm{D}_{2} \mathrm{O}$ at $298 \mathrm{~K}\left(e^{2} Q q / h=254 \mathrm{kHz}\right.$ and $\eta=$ $0.135)^{13)}$ were employed to estimate the $\tau_{2 \mathrm{R}}$ values for the mixtures.

Figure 3 shows the $\tau_{2 R}$ values estimated for $\mathrm{D}_{2} \mathrm{O}$ and the acetonitrile- $\mathrm{D}_{2} \mathrm{O}$ mixtures at each temperature as a function of $x_{\mathrm{AN}}$. At each temperature, the $\tau_{2 \mathrm{R}}$ value increases with increasing $x_{\mathrm{AN}}$ from 0 to 0.1 . Then, the value moderately decreases with further increasing $x_{\mathrm{AN}}$ and dips at $x_{\mathrm{AN}}=0.8$. When the temperature is lowered from 308 to $278 \mathrm{~K}$, the feature of the $\tau_{2 \mathrm{R}}$ becomes clearer; the increase in the $\tau_{2 \mathrm{R}}$ values in the range of $0.1 \leq x_{\mathrm{AN}} \leq 0.7$ with decreasing temperature is more significant than those at $x_{\mathrm{AN}}=0$ and 0.8 . It is thus suggested that the rotational motion of $\mathrm{D}_{2} \mathrm{O}$ molecules in the mixtures in the range of $0.1 \leq x_{\mathrm{AN}} \leq 0.7$ is more remarkably restricted by cooling than that at $x_{\mathrm{AN}}=0$ and 0.8 . The present results are comparable with those previously reported for acetonitrile- $\mathrm{D}_{2} \mathrm{O}$ mixtures at 283, 293, and $303 \mathrm{~K}^{14)}$ According to the phase diagram of acetonitrile-water mixtures, ${ }^{15)}{ }^{16)}$ the mole fraction range of $0.1 \leq x_{\mathrm{AN}} \leq 0.7$, where the $\tau_{2 \mathrm{R}}$ values significantly increases with decreasing temperature, agrees with that $\left(\sim 0.16<x_{\mathrm{AN}}<\sim 0.8\right)$ of phase separation of the mixtures by cooling. On the contrary, water ice is precipitated from the mixtures at $x_{\mathrm{AN}} \leq \sim 0.16$ and $x_{\mathrm{AN}} \geq \sim 0.8$.

This coincidence can be explained as follows. As shown in the previous large-angle X-ray scattering (LAXS) investigation on acetonitrile-water mixtures at $298 \mathrm{~K},{ }^{17)}$ water molecules aggregate with themselves by hydrogen bonding in the mixtures, while acetonitrile molecules interact by the dipoledipole interaction among them. However, acetonitrile and water molecules weakly interact with each other by the dipoledipole interaction between them. It results in the microinhomogeneity of acetonitrile-water mixtures in the range of $0.2<x_{\mathrm{AN}}<0.6$ even at the higher temperatures, as observed by the SANS experiments. When the temperature is lowered, water clusters formed are evolved in the mixtures toward phase separation because hydrogen bonds among water molecules are gradually strengthened. Thus, the rotational 

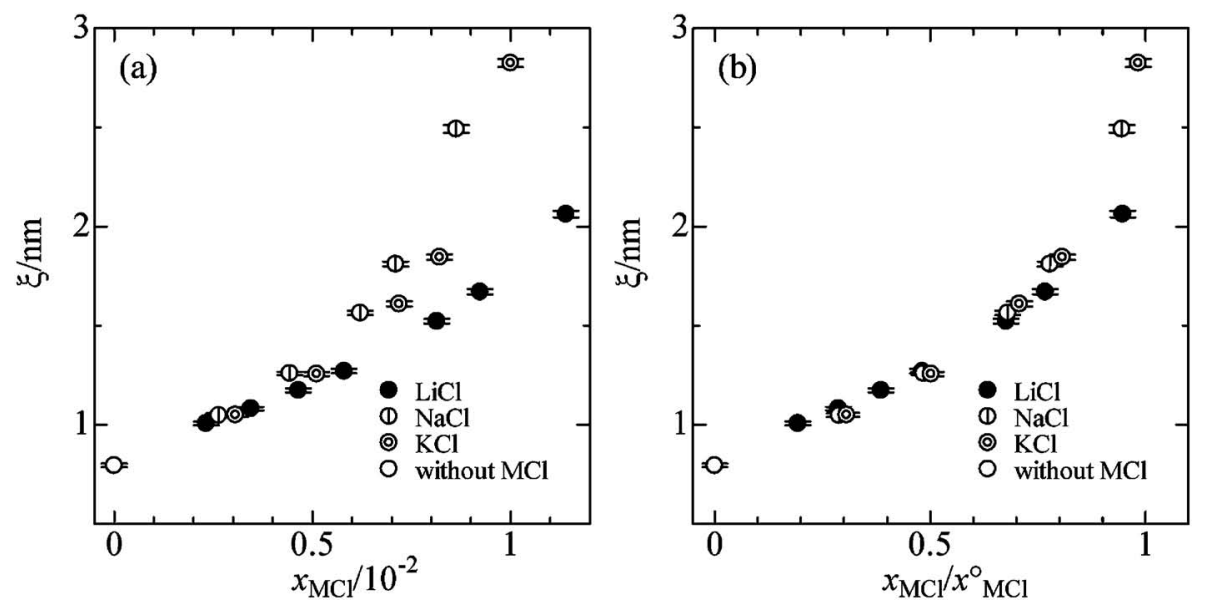

Fig. 4. Correlation lengths $\xi$ with the standard deviation of $\sigma$ values as error bars for acetonitrile- $\mathrm{D}_{2} \mathrm{O}-\mathrm{MCl}$ mixtures at $x_{\mathrm{AN}}=0.25$ as a function of (a) mole fraction of salt, $x_{\mathrm{MCl}}$, and (b) ratio of salt mole fraction to the critical one, $x_{\mathrm{MCl}}^{\circ}{ }^{18)}$

motion of water molecules in the mixtures at $0.1 \leq x_{\mathrm{AN}} \leq 0.7$ is strongly retarded with decreasing temperature. In the range of $x_{\mathrm{AN}}<0.1$, the hydrogen-bonded structure of water predominates in the mixtures even at the higher temperatures. On the other hand, in the range of $x_{\mathrm{AN}} \geq \sim 0.8$, water molecules may exist mainly as monomers in the inherent acetonitrile structure. Hence, the rotational motion of water molecules in the mixtures in both $x_{\mathrm{AN}}$-ranges is insensitive to the decrease in temperature.

\subsection{Acetonitrile- $\mathrm{D}_{2} \mathrm{O}-\mathrm{MCl}$ mixtures}

Even at $298 \mathrm{~K}$, phase separation of acetonitrile-water mixtures occurs by addition of salts, such as alkali chlorides, $\mathrm{MCl}$. The mechanism of salt-induced phase separation of the mixtures can be considered as follows. With increasing salt concentration, water clusters are gradually evolved around alkali metal and chloride ions in acetonitrile-water mixtures because of preferential solvation of the ions by water molecules due to the higher electron-donicity and acceptability of water molecule than acetonitrile one. In this process, acetonitrile clusters are gradually excluded from water clusters. When acetonitrile clusters and water clusters are grown to the macroscopic scale, finally, phase separation takes place. The SANS experiments enabled us to observe the enhancement of the microinhomogeneity for the acetonitrile- $\mathrm{D}_{2} \mathrm{O}-\mathrm{MCl}$ mixtures with increasing salt concentration. $\left..^{9)}, 18\right)$

In Fig. 4(a), the $\xi$ values determined for the acetonitrile$\mathrm{D}_{2} \mathrm{O}-\mathrm{MCl}$ mixtures at $x_{\mathrm{AN}}=0.25$ from the SANS experiments are plotted as a function of $\mathrm{MCl}$ mole fraction $x_{\mathrm{MCl}} \cdot{ }^{18)}$ For all the mixtures, the $\xi$ values increase with increasing $\mathrm{MCl}$ concentration, suggesting that water clusters and acetonitrile clusters are evolved in the mixtures. This is consistent with the mechanism proposed above. Figure 4 (a) also shows that the increase in the $\xi$ values with increasing $\mathrm{MCl}$ concentration is more significant in the order of $\mathrm{NaCl}>\mathrm{KCl}>\mathrm{LiCl}$. Actually, $\mathrm{MCl}$-induced phase separation of acetonitrile-water mixtures more easily occurs in this order. ${ }^{18)}$ However, the order is not in agreement with the expectation from the mechanism because of the stronger hydration of alkali metal ions in the order of $\mathrm{Li}^{+}>\mathrm{Na}^{+}>\mathrm{K}^{+}$as seen from the enthalpies of hydration of the ions $\left(-531,-416\right.$, and $-334 \mathrm{~kJ} \mathrm{~mol}^{-1}$, respectively). ${ }^{19)}$ In Fig. 4(b), accordingly, the $\xi$ values for the acetonitrile$\mathrm{D}_{2} \mathrm{O}-\mathrm{MCl}$ mixtures at $x_{\mathrm{AN}}=0.25$ are re-plotted as a function of $x_{\mathrm{MCl}} / x_{\mathrm{MCl}}^{\circ}$, where $x_{\mathrm{MCl}}^{\circ}$ is the critical $\mathrm{MCl}$ concentration required for phase separation $\left(x_{\mathrm{LiCl}}^{\circ}=1.20 \times 10^{-2}, x_{\mathrm{NaCl}}^{\circ}=\right.$
$0.913 \times 10^{-2}$, and $\left.x_{\mathrm{KCl}}^{\circ}=1.02 \times 10^{-2}\right) .{ }^{18)}$ Thus, this figure means that phase separation takes place when the $x_{\mathrm{MCl}} / x_{\mathrm{MCl}}^{\circ}$ reaches unity. As seen, the $\xi$ values for all the mixtures against the $x_{\mathrm{MCl}} / x_{\mathrm{MCl}}^{\circ}$ are overlapped. It is suggested that the microinhomogeneity of the mixtures is enhanced with increasing $\mathrm{MCl}$ concentration in the same way among all the $\mathrm{MCl}$ systems. Thus, the reason why LiCl-induced phase separation of the acetonitrile-water mixtures requires the highest salt concentration among the systems may be the rigid hydration structure of $\mathrm{Li}^{+}$due to the very strong electrostatic force. ${ }^{20)}$ It is likely that water molecules cannot readily orientate to form hydrogen bonds around the hydration shell of $\mathrm{Li}^{+}$. On the other hand, the hydrogen-bonded structure of water may not be markedly disrupted around the hydration shell of $\mathrm{K}^{+}$because $\mathrm{K}^{+}$is most loosely hydrated by water molecules among the alkali metal ions. The hydration shell of $\mathrm{Na}^{+}$is the most suitable to evolve water cluster due to the enough electrostatic interaction between $\mathrm{Na}^{+}$and water molecules.

Hence, the enhancement of water clusters in the mixtures is governed by the balance of the two opposite factors. One is the electrostatic force of the alkali metal ions to attract water molecules; it is stronger in the order of $\mathrm{Li}^{+}>\mathrm{Na}^{+}>\mathrm{K}^{+}$. The other is the formation of hydrogen bonds among water molecules around the hydration shell of the ions; it is easier in the order of $\mathrm{K}^{+}>\mathrm{Na}^{+}>\mathrm{Li}^{+}$. In consequence, phase separation of acetonitrile-water mixtures is most easily induced by $\mathrm{NaCl}$ among the $\mathrm{MCl}$ systems.

\subsection{1,4-Dioxane- $\mathrm{D}_{2} \mathrm{O}-\mathrm{NaCl}$ mixtures}

At ambient temperatures, 1,4-dioxane molecule, which involves two oxygen atoms as a hydrogen bond acceptor, is miscible with water one at any ratio. However, the previous SANS experiments on 1,4-dioxane- $\mathrm{D}_{2} \mathrm{O}$ mixtures showed that 1,4-dioxane and water molecules are not homogeneously mixed each other at the molecular level, although the microinhomogeneity of 1,4-dioxane-water mixtures is less significant than acetonitrile-water mixtures due to hydrogen bonds between 1,4-dioxane and water molecules. ${ }^{21), 22)}$ Furthermore, phase separation of 1,4-dioxane-water mixtures occurs by addition of salt as well as acetonitrile-water mixtures. ${ }^{21)}$ At the volume ratio $1: 1$ of the organic solvents to water, the critical $\mathrm{NaCl}$ concentration $\left(x_{\mathrm{NaCl}}^{\circ}=4.3 \times 10^{-2}\right)$ required for phase separation of the 1,4-dioxane- $\mathrm{D}_{2} \mathrm{O}$ mixture at $x_{\text {dio }}=$ 0.17 is much larger than that $\left(x_{\mathrm{NaCl}}^{\circ}=0.913 \times 10^{-2}\right)$ of the acetonitrile- $\mathrm{D}_{2} \mathrm{O}$ mixture at $x_{\mathrm{AN}}=0.25$. To clarify the enhance- 

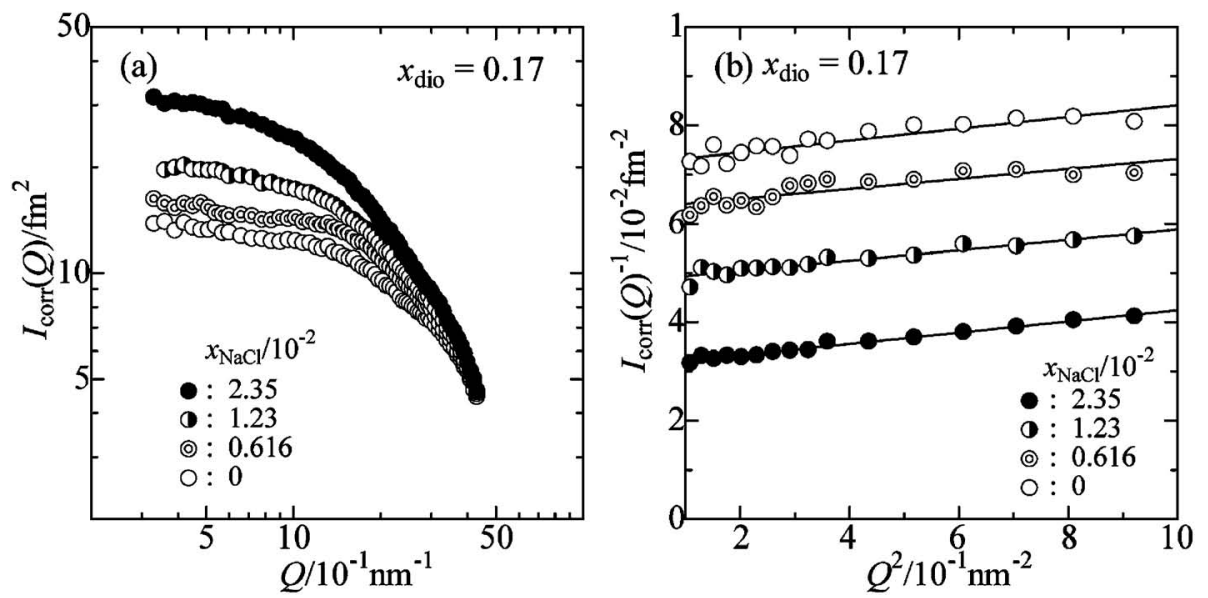

Fig. 5. (a) Normalized SANS intensities for 1,4 -dioxane- $\mathrm{D}_{2} \mathrm{O}-\mathrm{NaCl}$ mixtures at $x_{\text {dio }}=0.17$ as a function of $\mathrm{NaCl}$ concentration ${ }^{21)}$ and (b) Ornstein-Zernike plots. Circles give the experimental values and solid lines the theoretical ones.

ment of the microinhomogeneity for the 1,4-dioxane-water mixture at $x_{\text {dio }}=0.17$ with increasing salt concentration, SANS experiments were made on the mixture as a function of $\mathrm{NaCl}$ concentration. ${ }^{21)}$

Figure 5 (a) reveals the normalized SANS intensities of the 1,4-dioxane- $\mathrm{D}_{2} \mathrm{O}-\mathrm{NaCl}$ mixture at $x_{\text {dio }}=0.17$ measured on the WINK as a function of $x_{\mathrm{NaCl}}{ }^{21)}$ The SANS intensities of the mixtures are gradually strengthened with increasing $\mathrm{NaCl}$ concentration, showing that the microinhomogeneity of the mixtures is enhanced when the $\mathrm{NaCl}$ concentration increases. To quantitatively analyze the SANS intensities, the OrnsteinZernike plots were made on the reciprocal intensities as a function of the $Q^{2}$ value, as depicted in Fig. 5(b). Although the microinhomogeneity of the mixtures was evaluated in terms of the Debye correlation length $L_{\mathrm{D}}$ in the previous investigation, ${ }^{21)}$ the Ornstein-Zernike correlation length $\xi$ is applied to directly compare with that of the acetonitrile-water mixtures in the present investigation.

In Fig. 6, the $\xi$ values determined for the 1,4-dioxane- $\mathrm{D}_{2} \mathrm{O}-$ $\mathrm{NaCl}$ mixtures are depicted as a function of $\mathrm{NaCl}$ concentration. The upper axis represents the $\mathrm{NaCl}$ mole fraction $x_{\mathrm{NaCl}}$, whereas the lower one gives the ratio $x_{\mathrm{NaCl}} / x_{\mathrm{NaCl}}^{\circ}$ of $\mathrm{NaCl}$ concentration to the critical one required for phase separation $\left(x^{\circ}{ }_{\mathrm{NaCl}}=4.3 \times 10^{-2}\right)$. A comparison between Figs. 4 and 6 shows that the behavior of the $\xi$ values for the 1,4-dioxane$\mathrm{D}_{2} \mathrm{O}-\mathrm{NaCl}$ mixtures is different from that for the acetonitrile$\mathrm{D}_{2} \mathrm{O}-\mathrm{NaCl}$ mixtures. The $\xi$ values for the former are almost plateau in the range from $x_{\mathrm{NaCl}} / x_{\mathrm{NaCl}}^{\circ}=0$ to 0.2 and then significantly increase when the $\mathrm{NaCl}$ concentration further increases. On the contrary, those for the latter monotonously increase with increasing $\mathrm{NaCl}$ concentration toward $x_{\mathrm{NaCl}} /$ $x^{\circ}{ }_{\mathrm{NaCl}}=1$. This difference may arise from the degree of enhancement of water clusters. Hence, water clusters around the hydrated $\mathrm{Na}^{+}$and $\mathrm{Cl}^{-}$might be less easily formed in the 1,4dioxane-water mixtures than in the acetonitrile-water one because 1,4-dioxane molecule may form hydrogen bonds with water ones. On the other hand, water clusters around the hydration shells of $\mathrm{Na}^{+}$and $\mathrm{Cl}^{-}$could be easily formed in the acetonitrile-water mixtures because of the weak dipole-dipole interaction between water and acetonitrile molecules. Thus, $\mathrm{NaCl}$-induced phase separation of the 1,4-dioxane-water mixture requires the higher salt concentration than that of the acetonitrile-water mixture.

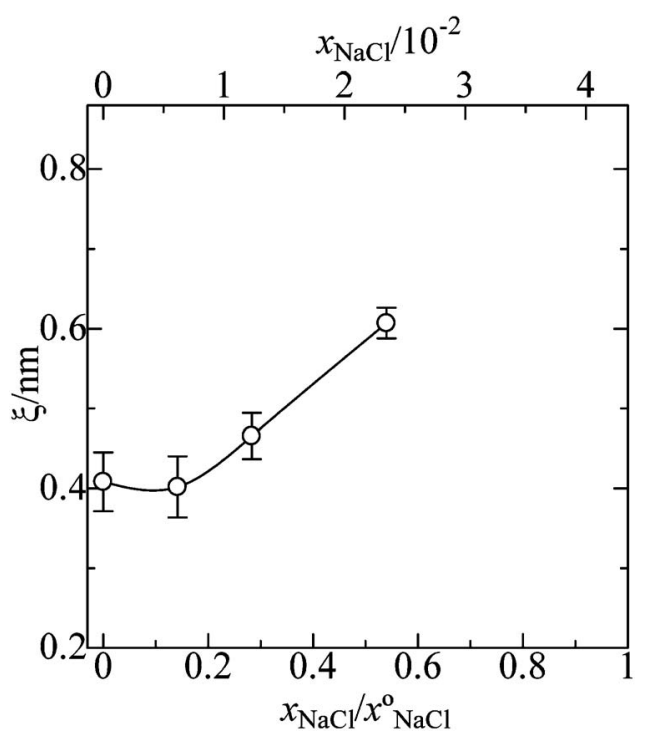

Fig. 6. Correlation lengths $\xi$ with the standard deviation of $\sigma$ values as error bars for 1,4-dioxane- $\mathrm{D}_{2} \mathrm{O}-\mathrm{NaCl}$ mixtures as a function of $\mathrm{NaCl}$ mole fraction; the upper axis represents $x_{\mathrm{NaCl}}$, and the lower axis gives $x_{\mathrm{NaCl}} / x_{\mathrm{NaCl}}^{\circ}$.

\subsection{Effect of microinhomogeneity for solvent mixtures on chemical reactions}

Acetonitrile-water and 1,4-dioxane-water mixtures are frequently used as an eluent for high performance liquid chromatography (HPLC). Based on the present results, the microinhomogeneity of both mixtures may play an important role on the mechanism of extraction process in HPLC. Probably, eluate is distributed between organic solvent clusters and water clusters formed in the mixtures as an eluent. The eluate may prefer to exist in organic solvent clusters, if it is a hydrophobic molecule, i.e., the concentration of the eluate in organic solvent clusters gradually increases during the travel of the eluate toward the end of HPLC column. Hence, various eluates including in a sample can be fractionated by HPLC depending on their hydrophobicity.

Aqueous mixtures of organic solvents, such as ethanol and acetonitrile, are applied to liquid phase synthesis of ceramic particle and powders like spherical silica. ${ }^{23)}$ The microin- 
homogeneity of solvent mixtures may influence the size and the dispersibility of silica spheres synthesized. The silica spheres are easily surrounded by water clusters rather than organic solvent clusters due to the hydrophilicity of silica surface. It is thus likely that the size of the silica spheres depends on that of water clusters formed in solvent mixtures. The monodispersibility of silica spheres will be enhanced in solvent mixtures, when the size of water clusters formed is uniform.

\section{Conclusion}

The SANS experiments on both acetonitrile- $\mathrm{D}_{2} \mathrm{O}$ and 1,4dioxane- $\mathrm{D}_{2} \mathrm{O}$ mixtures revealed that the organic molecules and water molecules are inhomogeneously mixed to form both clusters in the mixtures at the molecular level. The microinhomogeneity of both mixtures depends on the interaction between the organic molecules and water ones. In the acetonitrile-water mixtures, acetonitrile molecules mainly interact with water ones by the weak dipole-dipole interaction. On the other hand, 1,4-dioxane molecules may form hydrogen bonds with water ones. Thus, the microinhomogeneity of 1,4dioxane-water mixtures is less significant than that of acetonitrile-water ones. This is reflected to the fact that $\mathrm{NaCl}-$ induced phase separation of the 1,4-dioxane-water mixture less easily occurs than that of the acetonitrile-water mixture.

Acknowledgements This work was supported partly by Grants-in-Aid (No. 15550016 and 19550022) from the Ministry of Education, Culture, Sports, Science, and Technology, Japan. The SANS experiments were carried out by the joint researches of KENS, KEK. The NMR experiments were made at Division of Instrumental Analysis in Analytical Research Center for Experimental Sciences of Saga University.

\section{References}

1) F. Franks and D. J. G. Ives, Q. Rev. Chem. Soc., 20, 1-44 (1966).

2) F. Franks, "Water: A Comprehensive Treaties Vol. 2", Plenum Press, New York (1973).

3) T. Takamuku, D. Matsuo, A. Yamaguchi, M. Tabata, K. Yoshida, T. Yamaguchi, M. Nagao, T. Otomo and T. Adachi, Chem. Lett., 2000, 878-879.

4) A. Abragam, "The Principles of Nuclear Magnetism", Oxford University, Oxford (1961).
5) T. Otomo, M. Furusaka, S. Satoh, S. Itoh, T. Adachi, S. Shimizu and M. Takeda, J. Phys. Chem. Solids, 60, 1579-1582 (1999).

6) M. Furusaka, K.Suzuya, N. Watanabe, M. Osawa, I. Fujikawa and S. Satoh, KENS Report-IX, 25-27 (1992).

7) K. Yoshida, M. Misawa, K. Maruyama, M. Imai and M. Furusaka J. Chem. Phys., 113, 2343-2348 (2000).

8) V. F. Sears, "Thermal-Neutron Scattering Lengths and Cross Sections for Condensed-Matter Research", Chalk River Lab, Ontario (1984).

9) T. Takamuku, A. Yamaguchi, D. Matsuo, M. Tabata, M. Kumamoto, J. Nishimoto, K. Yoshida, T. Yamaguchi, M. Nagao, T. Otomo and T. Adachi, J. Phys. Chem. B, 105, 6236-6245 (2001).

10) T. Takamuku, Y. Noguchi, M. Matsugami, H. Iwase, T. Otomo and M. Nagao, J. Mol. Liquids, 136, 147-155 (2007).

11) H. E. Stanley, "Introduction to Phase Transitions and Critical Phenomena," Oxford University Press, Oxford (1971).

12) D. A. Armitage, M. J. Blandamer, M. J. Foster, N. J. Hidden, K. W. Morcom, M. C. R. Symons, M. J. Wootten, J. Trans. Faraday Soc., 64, 1193-1200 (1968).

13) R. P. W. J. Struis, J. De Bleijser, J. C. Leyte, J. Phys. Chem., 91, 1639-1645 (1987).

14) M. Ueno, S. Ueyama, S. Hashimoto, N. Tsuchihashi and K. Ibuki, J. Solution Chem., 33, 827-845 (2004).

15) G. Schneider, Z. Physik. Chem. N. F., 41, 327-338 (1964).

16) J. Szydlowski and M. Szykula, Fluid Phase Equilibria, 154, 79-87 (1999).

17) T. Takamuku, M. Tabata, M. Kumamoto, A. Yamaguchi, J. Nishimoto, H. Wakita and T. Yamaguchi, J. Phys. Chem. B, 102, 8880-8888 (1998).

18) T. Takamuku, Y. Noguchi, E. Yoshikawa, T. Kawaguchi, M. Matsugami and T. Otomo, J. Mol. Liquids, 131/132, 131-138 (2007).

19) Y. Marcus, J. Chem. Soc. Faraday Trans., 83, 339-349 (1987).

20) T. Yamaguchi, M. Yamagami, H Ohzono, K. Yamanaka and H. Wakita, Physica B 213/214, 480-482 (1995).

21) T. Takamuku, A. Yamaguchi, D. Matsuo, M. Tabata, T. Yamaguchi, T. Otomo and T. Adachi, J. Phys. Chem. B, 105, 10101-10110 (2001).

22) T. Takamuku, A. Nakamizo, M. Tabata, K. Yoshida, T. Yamaguchi and T. Otomo, J. Mol. Liquids, 103/104, 143-159 (2003).

23) N. Enomoto, A. Kumagai and J. Hoho, J. Ceram. Soc. Japan, 113, 340-343 (2005). 